\title{
Free lattices proof-theoretically
}

\author{
Tomasz Kowalski \\ Department of Mathematics and Statistics \\ La Trobe University \\ Melbourne, Australia
}

\begin{abstract}
A sequent system is used to give alternative proofs of two well known properties of free lattices: Whitman's condition and semidistributivity. It demonstrates usefulness of such proof systems outside logic.
\end{abstract}

\section{Introduction}

Sequent systems are naturally considered in relation to logics. The connection however seems to be accidental: nothing in the general concept of a sequent system is forcing it to be a proof system for a logic (except perhaps a trace of an ordering relation inherent in sequents). In this note, I will show that a natural sequent system can be used to obtain reasonably easy proofs of two important properties of free lattices. These properties are not new - by far the contrary - yet I think there is some merit to the exercise, especially in view of [4] where it is shown that no decent term rewriting system exists for lattices.

If logic is understood as abstract algebraic logic (in a broader sense than the original concept from [1]; see [2] for a recent comprehensive textbook), the class of all lattices turns out to be a (necessarily unique) equivalent algebraic semantics of the sequent system I will use, so there is a logic connection after all, if we are pressed hard enough to find it. But algebraizability and related notions will play no role in the proofs. The system itself is inspired by the sequent calculus used in $[14,15]$ for basic quantum logic (orthologic), but it uses multisets rather than sets.

\section{Preliminaries}

We assume familiarity with the basics of lattice theory and of sequent calculi. For a compendium of lattice theory the reader is referred to [5]; Chapter I and Appendix G will be especially useful in the present context. For sequent systems in logic and their connections to algebra, [16] is highly recommended. 
We use lowercase Greek letters for lattice terms, and lowercase Roman letters for elements of free lattices, i.e., equivalence classes of terms. When convenient we will match the Greek and Roman letters in the obvious way, so that $a=[\alpha], b=[\beta], c=[\gamma]$, etc.

Sequents are pairs of finite multisets ot terms. We use uppercase Greek letters for multisets of terms, and write sequents in the traditional $\Gamma \Rightarrow \Delta$ way, with $\Rightarrow$ as the separator. Moreover, we will write $\vdash \Gamma \Rightarrow \Delta$ as a shorthand for " $\Gamma \Rightarrow \Delta$ is provable" (in some sequent system clear from context). Furthermore, we will abbreviate " $\vdash \gamma \Rightarrow \delta$ and $\vdash \delta \Rightarrow \gamma^{\prime \prime}$ by " $\vdash \gamma \Leftrightarrow \delta^{\prime \prime}$.

Speaking about multisets in general, we will use the standard set-theoretical notation and rely on the reader to give it the appropriate interpretation. For example $\{\alpha, \beta\} \cup\{\alpha\}=$ $\{\alpha, \alpha, \beta\}$, and $\{\alpha, \alpha\} \neq\{\alpha\}$. For sequents, however, we will use the traditional commaseparated list notation, writing e.g., $\Gamma, \alpha, \alpha \Rightarrow \Delta$ rather than $\Gamma \cup\{\alpha, \alpha\} \Rightarrow \Delta$.

\section{A sequent system for lattices}

The initial sequents are:

$$
\alpha \Rightarrow \alpha
$$

Since we use multisets, the rule of exchange is built in, so as structural rules we only take weakening

$$
\frac{\Gamma \Rightarrow \Delta}{\alpha, \Gamma \Rightarrow \Delta} \quad \frac{\Gamma \Rightarrow \Delta}{\Gamma \Rightarrow \Delta, \alpha}
$$

contraction

$$
\frac{\alpha, \alpha, \Gamma \Rightarrow \Delta}{\alpha, \Gamma \Rightarrow \Delta} \quad \frac{\Gamma \Rightarrow \Delta, \alpha, \alpha}{\Gamma \Rightarrow \Delta, \alpha}
$$

and restricted cut

$$
\frac{\Gamma \Rightarrow \alpha, \Delta \quad \alpha \Rightarrow \Pi}{\Gamma \Rightarrow \Delta, \Pi} \quad \frac{\Gamma \Rightarrow \alpha \quad \Sigma, \alpha \Rightarrow \Pi}{\Gamma, \Sigma \Rightarrow \Pi}
$$

Unrestricted cut would make the system too strong. The rules for $\wedge$ and $\vee$ are

$$
\begin{array}{ccc}
\frac{\Gamma, \alpha \Rightarrow \Delta}{\Gamma, \alpha \wedge \beta \Rightarrow \Delta} & \frac{\Gamma, \beta \Rightarrow \Delta}{\Gamma, \alpha \wedge \beta \Rightarrow \Delta} & \frac{\Gamma \Rightarrow \alpha \quad \Gamma \Rightarrow \beta}{\Gamma \Rightarrow \alpha \wedge \beta} \\
\frac{\Gamma \Rightarrow \alpha, \Delta}{\Gamma \Rightarrow \alpha \vee \beta, \Delta} & \frac{\Gamma \Rightarrow \beta, \Delta}{\Gamma \Rightarrow \alpha \vee \beta, \Delta} & \frac{\alpha \Rightarrow \Delta \quad \beta \Rightarrow \Delta}{\alpha \vee \beta \Rightarrow \Delta}
\end{array}
$$

Allowing contexts on the right for $\wedge$-right, and on the left for $\vee$-left would make the system too strong.

We will call the sequent system defined above $\mathbf{L G}^{+}$. To illustrate a proof (two proofs, in fact) in $\mathbf{L G}^{+}$, we will show that $\vdash \alpha \wedge(\alpha \vee \beta) \Leftrightarrow \alpha$.

$$
\frac{\alpha \Rightarrow \alpha}{\alpha \wedge(\alpha \vee \beta) \Rightarrow \alpha} \quad \frac{\alpha \Rightarrow \alpha \frac{\alpha \Rightarrow \alpha}{\alpha \Rightarrow \alpha \vee \beta}}{\alpha \Rightarrow \alpha \wedge(\alpha \vee \beta)}
$$

Australasian Journal of Logic (17:2) 2020, Article no. 2 
Similarly, for any identity $\alpha \approx \beta$ in the standard basis for lattices, it is easy to show $\vdash \alpha \Leftrightarrow \beta$. An instructive exercise is to find cut-free proofs of the relevant sequents.

We should also make clear what we meant by "too strong" above. For example, using unrestricted cut, that is

$$
\frac{\Gamma \Rightarrow \alpha, \Delta \quad \Sigma, \alpha \Rightarrow \Pi}{\Gamma, \Sigma \Rightarrow \Delta, \Pi}
$$

we would have the following proof.

$$
\frac{\frac{\alpha \Rightarrow \alpha}{\alpha \Rightarrow \alpha, \alpha \wedge \beta} \quad \frac{\alpha \Rightarrow \alpha}{\beta \vee \gamma, \alpha \Rightarrow \alpha \vee \gamma}}{\alpha \wedge(\beta \vee \gamma) \Rightarrow(\alpha \wedge \beta) \vee(\alpha \vee \gamma)}
$$

which together with the (cut free) proof

$$
\begin{array}{ccc}
\frac{\alpha \Rightarrow \alpha}{\alpha \wedge \beta \Rightarrow \alpha} \frac{\beta \Rightarrow \beta}{\alpha \wedge \beta \Rightarrow \beta} & \frac{\gamma \Rightarrow \gamma}{\alpha \wedge \gamma \Rightarrow \beta} \\
\frac{\alpha \wedge \beta \Rightarrow \alpha \wedge(\beta \vee \gamma)}{\alpha \wedge \beta \vee \gamma} & \frac{\alpha \wedge \gamma \Rightarrow \alpha}{\alpha \wedge \gamma \Rightarrow \beta \vee \gamma} \\
\frac{\alpha \wedge \gamma \Rightarrow \alpha \wedge(\beta \vee \gamma)}{\alpha \wedge \beta)(\alpha \vee \gamma) \Rightarrow \alpha \wedge(\beta \vee \gamma)} & \frac{\alpha \wedge \gamma)}{\alpha \wedge(\beta \wedge \gamma)}
\end{array}
$$

would yield $\vdash \alpha \wedge(\beta \vee \gamma) \Leftrightarrow(\alpha \wedge \beta) \vee(\alpha \vee \gamma)$, a counterpart of the distributive identity. Strengthening the rules $\wedge$-right and $\vee$-left to

$$
\frac{\Gamma \Rightarrow \alpha, \Delta \quad \Gamma \Rightarrow \beta, \Delta}{\Gamma \Rightarrow \alpha \wedge \beta, \Delta} \quad \frac{\Gamma, \alpha \Rightarrow \Delta \quad \Gamma, \beta \Rightarrow \Delta}{\Gamma, \alpha \vee \beta \Rightarrow \Delta}
$$

would have the same effect. Hence the restrictions.

Although distributivity cannot be proved, the interpretation of comma in the sequents is the same as in classical logic (where of course distributivity is provable), namely, comma on the left is $\wedge$ and comma on the right is $\vee$. The next lemma makes it precise, but we introduce a shorthand first. For any multiset $\Gamma=\left\{\gamma_{1}, \ldots, \gamma_{n}\right\}$ of terms, we write $\bigwedge \Gamma$ for any parenthesising of $\gamma_{1} \wedge \cdots \wedge \gamma_{n}$. Similarly, $\bigvee \Gamma$ will stand for any parenthesising of $\gamma_{1} \vee \cdots \vee \gamma_{n}$. Since associativity of $\wedge$ and $\vee$ is provable, i.e., we have $\vdash \alpha \wedge(\beta \wedge \gamma) \Leftrightarrow$ $(\alpha \wedge \beta) \wedge \gamma$ and $\vdash \alpha \vee(\beta \vee \gamma) \Leftrightarrow(\alpha \vee \beta) \vee \gamma$, this piece of notation is harmless.

Lemma 1. Let $\Gamma$ and $\Delta$ be multisets of terms, and let $\alpha$ and $\beta$ be terms. The following hold:

1. $\vdash \Gamma \Rightarrow \alpha, \beta, \Delta$ if and only if $\vdash \Gamma \Rightarrow \alpha \vee \beta, \Delta$.

2. $\vdash \Gamma, \alpha, \beta \Rightarrow \Delta$ if and only if $\vdash \Gamma, \alpha \wedge \beta \Rightarrow \Delta$.

3. $\vdash \Gamma \Rightarrow \Delta$ if and only if $\vdash \wedge \Gamma \Rightarrow \bigvee \Delta$.

Australasian Journal of Logic (17:2) 2020, Article no. 2 
4. $\vdash \alpha \Rightarrow \beta$ if and only if $\vdash \alpha \Rightarrow \alpha \wedge \beta$.

5. $\vdash \alpha \Rightarrow \beta$ if and only if $\vdash \alpha \vee \beta \Rightarrow \beta$.

Proof. For the forward direction of (1), assume $\vdash \Gamma \Rightarrow \alpha, \beta, \Delta$. Then, the following proof

$$
\frac{\frac{\Gamma \Rightarrow \alpha, \beta, \Delta}{\Gamma \Rightarrow \alpha, \alpha \vee \beta, \Delta}}{\frac{\Gamma \Rightarrow \alpha \vee \beta, \alpha \vee \beta, \Delta}{\Gamma \Rightarrow \alpha \vee \beta, \Delta}}
$$

shows $\vdash \Gamma \Rightarrow \alpha \vee \beta, \Delta$. Note that it uses contraction. For the backward direction, assume $\vdash \Gamma \Rightarrow \alpha \vee \beta, \Delta$. Then, the following proof

$$
\frac{\frac{\alpha \Rightarrow \alpha}{\alpha \Rightarrow \alpha, \beta} \quad \frac{\beta \Rightarrow \beta}{\beta \Rightarrow \alpha, \beta}}{\alpha \vee \alpha \vee \beta, \Delta} \frac{\frac{\beta \Rightarrow \alpha}{\alpha \vee \beta}}{\Gamma \Rightarrow \alpha, \beta, \Delta}
$$

shows $\vdash \Gamma \Rightarrow \alpha, \beta, \Delta$. Note that it uses cut. The proof of (2) is dual, and (3) follows from (1) and (2). The proofs of (4) and (5) are very easy (using cut for the backward direction).

Now we have everything we need to prove that $\mathbf{L G}^{+}$is indeed a proof system for lattices (or, equivalently, for free lattices). The proof proceeds by an obvious variant of the standard Lindenbaum algebra construction and presents no difficulty, but it is included for completeness.

Theorem 1. The relation $\equiv$ on the absolutely free algebra $\mathbf{T}(\Xi)$ of lattice terms in variables from $\Xi$, defined by $\alpha \equiv \beta$ if $\vdash \alpha \Leftrightarrow \beta$ holds, is a fully invariant congruence. The quotient algebra $\mathbf{T}(\Xi) / \equiv$ is the free lattice generated by $\Xi / \equiv$.

Proof. The first statement is proved by the standard Lindenbaum argument. In particular, transitivity of $\equiv$ follows by cut, and being fully invariant follows from closure of the sequent rules under substitution. It follows that $\mathbf{T}(\Xi) / \equiv$ is free in some variety $\mathcal{V}$, and verification of the fact that $\mathbf{T}(\Xi) / \equiv$ is a lattice presents no difficulty. In particular, the relation $[\alpha] \leqslant[\beta]$ defined by $\vdash \alpha \Rightarrow \beta$ is the lattice order on $\mathbf{T}(\Xi)$.

It remains to show that $\mathcal{V}$ is the variety of all lattices. It suffices to show that for any finite multisets of of terms $\Gamma$ and $\Delta$ we have that $\vdash \Gamma \Rightarrow \Delta$ implies that the inequality $\bigwedge \Gamma \leqslant \bigvee \Delta$ holds in all lattices. This can be done by induction on the length of proof of $\Gamma \Rightarrow \Delta$. If $\Gamma \Rightarrow \Delta$ is an initial sequent, the claim is trivially true. Consider the last rule in the proof of $\Gamma \Rightarrow \Delta$, say, for example, that it was cut in the form

$$
\frac{\Gamma^{\prime} \Rightarrow \alpha \quad \Gamma^{\prime \prime}, \alpha \Rightarrow \Delta}{\Gamma \Rightarrow \Delta}
$$

Australasian Journal of Logic (17:2) 2020, Article no. 2 
where $\Gamma=\Gamma^{\prime}, \Gamma^{\prime \prime}$. By inductive hypothesis, we have that the inequalities $\wedge \Gamma^{\prime} \leqslant \alpha$ and $\wedge \Gamma^{\prime \prime} \wedge \alpha \leqslant \bigvee \Delta$ hold in all lattices. Then, $\wedge \Gamma^{\prime} \wedge \wedge \Gamma^{\prime \prime} \leqslant \wedge \Gamma^{\prime \prime} \wedge \alpha \leqslant \bigvee \Delta$, so $\wedge \Gamma^{\prime} \wedge \wedge \Gamma^{\prime \prime} \leqslant \bigvee \Delta$ holds, and so $\wedge \Gamma \leqslant \bigvee \Delta$ holds as required. (Note how this reasoning would fail for unrestricted cut.) Now repeating a similar argument for all other rules, we finish the proof.

If we carefully distinguished the equational language of lattices and the propositional language of the sequent system, the proof above (plus a few technical details) will show that the class of all lattices is the equivalent algebraic semantics for $\mathbf{L G}^{+}$, in the extended sense of algebraizability for which no equivalence connective is necessary (see [2]). We will not enter into further details, since algebraizability is not directly relevant for our purposes.

The next two lemmas state some technicalities that will be of use in Section 5 .

Lemma 2. Let $\Gamma$ and $\Delta$ be multisets of terms, and let $\alpha$ and $\beta$ be terms. If $\vdash \Gamma \Rightarrow \Delta$ and $\vdash \alpha \Leftrightarrow \beta$ hold, then $\vdash \Gamma[\alpha / \beta] \Rightarrow \Delta[\alpha / \beta]$ holds, where $\Gamma[\alpha / \beta]$ and $\Delta[\alpha / \beta]$ are the results of replacing some occurrences of a subterm $\alpha$ in some members of $\Gamma$ and $\Delta$, by $\beta$.

Proof. Induction on the length of the proof of $\Gamma \Rightarrow \Delta$.

Let $\tau$ be a lattice term. We define multisets $L^{b}(\tau)$ and $R^{b}(\tau)$ recursively as follows.

- If $\tau$ is a variable, then $L^{b}(\tau)=\{\tau\}=R^{b}(\tau)$.

- If $\tau=\tau_{1} \wedge \tau_{2}$, then $L^{b}(\tau)=L^{b}\left(\tau_{1}\right) \cup L^{b}\left(\tau_{2}\right)$ and $R^{b}(\tau)=\{\tau\}$.

- If $\tau=\tau_{1} \vee \tau_{2}$, then $L^{b}(\tau)=\{\tau\}$ and $R^{b}(\tau)=R^{b}\left(\tau_{1}\right) \cup R^{b}\left(\tau_{2}\right)$.

Lemma 3. Let $\Gamma, \Delta$ be multisets of terms, and let $\tau$ be a term. Then

- $\vdash \tau, \Gamma \Rightarrow \Delta$ if and only if $\vdash L^{b}(\tau), \Gamma \Rightarrow \Delta$,

- $\vdash \Gamma \Rightarrow \Delta$, $\tau$ if and only if $\vdash \Gamma \Rightarrow \Delta, R^{b}(\tau)$.

In particular, we have $\vdash L^{b}(\tau) \Rightarrow \tau$ and $\vdash \tau \Rightarrow R^{b}(\tau)$.

Proof. By induction and Lemma 1.

\section{Cut and contraction elimination}

Let $\mathbf{L G}$ be the sequent system $\mathbf{L G}^{+}$without the rule of cut. Using completely standard methods (see, e.g., [16]), it is not difficult to prove the following cut elimination result.

Lemma 4. The following are equivalent:

1. $\Gamma \Rightarrow \Delta$ is provable in $\mathbf{L G}^{+}$.

Australasian Journal of Logic (17:2) 2020, Article no. 2 


\section{2. $\Gamma \Rightarrow \Delta$ is provable in $\mathbf{L G}$.}

Interestingly, contraction also turns out to be eliminable, albeit from a slightly modified system. Let $\mathbf{L} \mathbf{G}^{\sharp}$ be the system obtained from $\mathbf{L G}^{+}$by replacing the left $\wedge$-introduction and righ $\vee$-introduction rules, by their versions below:

$$
\frac{\Gamma, \alpha, \beta \Rightarrow \Delta}{\Gamma, \alpha \wedge \beta \Rightarrow \Delta} \quad \frac{\Gamma \Rightarrow \alpha, \beta, \Delta}{\Gamma \Rightarrow \alpha \vee \beta, \Delta}
$$

Note that contraction is derivable in $\mathbf{L G}^{\sharp}$, using cut. Here is a proof of left-side contraction

$$
\frac{\frac{\alpha \Rightarrow \alpha \quad \alpha \Rightarrow \alpha}{\alpha \Rightarrow \alpha \wedge \alpha} \quad \frac{\Gamma, \alpha, \alpha \Rightarrow \Delta}{\Gamma, \alpha \wedge \alpha \Rightarrow \Delta}}{\Gamma, \alpha \Rightarrow \Delta}
$$

and right-side contraction can be derived analogously. Conversely, the modified rules of $\mathbf{L G}^{\sharp}$ are derivable by contraction. Right $\vee$-introduction can be derived by

$$
\frac{\frac{\Gamma \Rightarrow \alpha, \beta, \Delta}{\Gamma \Rightarrow \alpha \vee \beta, \beta, \Delta}}{\frac{\Gamma \Rightarrow \alpha \vee \beta, \alpha \vee \beta, \Delta}{\Gamma \Rightarrow \alpha \vee \beta, \Delta}}
$$

and left $\wedge$-introduction analogously. Therefore, sytems LG and $\mathbf{L G}^{\sharp}$ are equivalent, in the sense that a sequent is derivable in one if and only if it is derivable in the other. Cut elimination theorem holds for $\mathbf{L G}^{\sharp}$ as well. In fact, it is easier to prove for $\mathbf{L G}^{\sharp}$ than for LG. For $\mathbf{L G}^{\sharp}$ does not have contraction, so we do not need to replace cut by mix to hide it. Let $\mathbf{L G}^{b}$ be the system obtained from $\mathbf{L G}^{\sharp}$ by removing cut. The following theorem summarises the section.

Theorem 2. The following are equivalent:

1. $\Gamma \Rightarrow \Delta$ is provable in $\mathbf{L G}^{+}$.

2. $\Gamma \Rightarrow \Delta$ is provable in $\mathbf{L G}$.

3. $\Gamma \Rightarrow \Delta$ is provable in $\mathbf{L G}^{\sharp}$.

4. $\Gamma \Rightarrow \Delta$ is provable in $\mathbf{L G}^{b}$.

From now on, LG will be our official proof system. In particular, $\vdash \Gamma \Rightarrow \Delta$ will mean that $\Gamma \Rightarrow \Delta$ is provable in $\mathbf{L G}$. 


\section{Whitman's condition}

The following property was isolated by Whitman in his proof of decidability of the word problem for free lattices.

(W) If $a=a_{1} \wedge \cdots \wedge a_{n} \leqslant b_{1} \vee \cdots \vee b_{m}=b$, then $a_{i} \leqslant b$ for some $i$, or $a \leqslant b_{j}$ for some $j$.

Whitman proved that $(\mathrm{W})$ holds in free lattices. The property is now known as Whitman's condition and has been widely studied. We will use LG to give an easy proof. First, we introduce a piece of terminology. For multisets of terms $\Gamma$ and $\Delta$ we will say that the sequent $\Gamma \Rightarrow \Delta$ is minimally provable (written $\vdash_{\min } \Gamma \Rightarrow \Delta$ ), if $\vdash \Gamma \Rightarrow \Delta$ and for any $\Gamma^{\prime} \subseteq \Gamma$ and $\Delta^{\prime} \subseteq \Delta$ such that at least one of the multiset inclusions is proper, $\nvdash \Gamma^{\prime} \Rightarrow \Delta^{\prime}$.

Lemma 5. Let $\Gamma=\left\{\gamma_{1}, \ldots, \gamma_{n}\right\}$ and $\Delta=\left\{\delta_{1}, \ldots, \delta_{m}\right\}$ be multisets of terms, satisfying the following conditions:

1. $\vdash_{\min } \Gamma \Rightarrow \Delta$.

2. If $n=1$, then $R^{b}\left(\gamma_{1}\right)=\gamma_{1}$.

3. If $m=1$, then $L^{b}\left(\delta_{1}\right)=\delta_{1}$.

Then $\vdash \Gamma \Rightarrow \Delta$ if and only if $\vdash \gamma_{i} \Rightarrow \delta_{j}$ for some $i \in\{1, \ldots, n\}$ and some $j \in\{1, \ldots, m\}$.

Proof. The conditions (2) and (3) state that if $\Gamma$ is a singleton, then its single member is not a join, and if $\Delta$ is a singleton, its single member is not a meet. Then the result follows by easy induction on the length of cut-free proof of $\Gamma \Rightarrow \Delta$ (observe that weakening and two-premiss rules never apply).

By finiteness and weakening it is easily shown that $\vdash \Gamma \Rightarrow \Delta$ holds if and only if $\vdash_{\min } \Gamma^{\prime} \Rightarrow \Delta^{\prime}$ holds for some $\Gamma^{\prime} \subseteq \Gamma$ and $\Delta^{\prime} \subseteq \Delta$.

Lemma 6. Let $\Gamma=\left\{\gamma_{1}, \ldots, \gamma_{n}\right\}$ and $\Delta=\left\{\delta_{1}, \ldots, \delta_{m}\right\}$ be multisets of terms. Then $\vdash \Gamma \Rightarrow \Delta$ if and only if $\vdash \Gamma \Rightarrow \delta_{j}$ for some $j \in\{1, \ldots, m\}$ or $\vdash \gamma_{i} \Rightarrow \Delta$ for some $i \in\{1, \ldots, n\}$.

Proof. The backward direction is trivial. The forward direction is proved by induction on complexity of $\Gamma \Rightarrow \Delta$. If $\Gamma$ or $\Delta$ is a singleton, then the claim holds trivially. If neither is a singleton, then by inductive hypothesis we can assume $\vdash_{\min } \Gamma \Rightarrow \Delta$. Then, (2) and (3) of Lemma 5 are satisfied, and the claim follows.

Theorem 3. The property $(W)$ holds in free lattices.

Proof. By Lemma 6.

Australasian Journal of Logic (17:2) 2020, Article no. 2 


\section{A naive decision algorithm}

We mentioned at the beginning of the section that Whitman used (W) to prove decidability of the word problem for free lattices. We can get a quick and easy decidability proof directly from the sequent system. For the inequality $\alpha \leqslant \beta$ holds in free lattices if and only if the sequent $\alpha \Rightarrow \beta$ is provable in $\mathbf{L G}^{b}$. A naive decision algorithm is just to construct all possible proof trees for $\alpha \Rightarrow \beta$. Since in all rules of $\mathbf{L G}^{b}$ the upper sequents are simpler than the lower sequents, the construction terminates, and $\alpha \Rightarrow \beta$ is provable if and only if there is a proof tree whose all leaves are initial sequents. The complexity of the naive decision algorithm is however exponential. The best known decision algorithm (see [3]) is of quadratic time complexity. It will be interesting to refine the naive sequent system algorithm and reduce the complexity, but this is beyond the scope of the present note.

\section{Semidistributivity}

The following quasi-identities:

$$
\begin{aligned}
& x \vee y=x \vee z \quad \longrightarrow \quad x \vee y=x \vee(y \wedge z) \\
& x \wedge y=x \wedge z \quad \longrightarrow \quad x \wedge y=x \wedge(y \vee z)
\end{aligned}
$$

are known as that join semidistributivity $\left(\mathrm{SD}_{\vee}\right)$ and meet semidistributivity $\left(\mathrm{SD}_{\wedge}\right)$. A lattice is called semidistributive if it satisfies both $\left(\mathrm{SD}_{\wedge}\right)$ and $\left(\mathrm{SD}_{\vee}\right)$. Jónsson and Kiefer showed that free lattices are semidistributive. We will use LG to give a proof, which although not quite easy, is simpler than the original. The next lemma can be seen as a variant of Lemma 5.

Lemma 7. Let $\Gamma=\left\{\gamma_{1}, \ldots, \gamma_{n}\right\}$ and $\Delta=\left\{\delta_{1}, \ldots, \delta_{m}\right\}$ be multisets of terms.

1. If $n=1$ and $R^{b}\left(\gamma_{1}\right)=\gamma_{1}$, then $\vdash \gamma_{1} \Rightarrow \Delta$ if and only if $\vdash \gamma_{1} \Rightarrow \delta_{j}$ for some $j \in\{1, \ldots, m\}$.

2. If $m=1$ and $L^{b}\left(\delta_{1}\right)=\delta_{1}$, then $\vdash \Gamma \Rightarrow \delta_{1}$ if and only if $\vdash \gamma_{i} \Rightarrow \delta_{1}$ for some $i \in\{1, \ldots, n\}$.

Proof. Only the forward directions are non-trivial, and since (1) and (2) are dual, it suffices to prove the forward direction of (1). To this end, assume $R^{b}\left(\gamma_{1}\right)=\gamma_{1}$ and $\vdash \gamma_{1} \Rightarrow \Delta$. If $\vdash_{\min } \gamma_{1} \Rightarrow \Delta$, the claim follows by Lemma 5. If $\gamma_{1} \Rightarrow \Delta$ is not minimally provable, then let $\Delta^{\prime}$ be a submultiset of $\Delta$ such that $\vdash_{\min } \gamma_{1} \Rightarrow \Delta^{\prime}$ holds. Then, Lemma 5 applies, and so $\vdash \gamma \Rightarrow \delta_{j}$ holds for some $\delta_{j} \in \Delta^{\prime} \subset \Delta$, thus the claim follows as well.

Lemma 8. Let $\alpha, \beta, \gamma$ be lattice terms. If $R^{b}(\beta)=\beta$ or $R^{b}(\gamma)=\gamma$, and $\beta \Rightarrow \alpha, \gamma$ and $\gamma \Rightarrow \alpha, \beta$ are provable, then $\beta \Rightarrow \alpha, \beta \wedge \gamma$ is provable as well. 
Proof. Assume $R^{b}(\beta)=\beta$. Then by Lemma 5 we have $\vdash \beta \Rightarrow \alpha$ or $\vdash \beta \Rightarrow \gamma$. If $\vdash \beta \Rightarrow \alpha$, then $\vdash \beta \Rightarrow \alpha, \beta \wedge \gamma$ by weakening. If $\vdash \beta \Rightarrow \gamma$, then $\vdash \beta \Rightarrow \beta \wedge \gamma$ as well, and again by weakening $\vdash \beta \Rightarrow \alpha, \beta \wedge \gamma$.

Next, assume $R^{b}(\gamma)=\gamma$. Then by Lemma 7 we have $1 \vdash \gamma \Rightarrow \alpha$ or $\vdash \gamma \Rightarrow \beta$. Now, if $\vdash \gamma \Rightarrow \alpha$, then as $\vdash \beta \Rightarrow \alpha, \gamma$ by assumption, we get $\vdash \beta \Rightarrow \alpha$ by cut and contraction. Thus, $\vdash \beta \Rightarrow \alpha, \beta \wedge \gamma$. Finally, if $\vdash \gamma \Rightarrow \beta$, then $\vdash \gamma \Rightarrow \beta \wedge \gamma$ as well, and then by cut $\vdash \beta \Rightarrow \alpha, \beta \wedge \gamma$.

Lemma 9. Let $\alpha, \beta, \gamma$ be lattice terms. If $\beta \Rightarrow \alpha, \gamma$ and $\gamma \Rightarrow \alpha, \beta$ are provable, then so is $\beta \Rightarrow \alpha, \beta \wedge \gamma$.

Proof. Let $R^{b}(\beta)=\left\{\beta_{1}, \ldots, \beta_{n}\right\}$ and $R^{b}(\gamma)=\left\{\gamma_{1}, \ldots, \gamma_{m}\right\}$. Spelt out fully, these assumptions mean that (i) $\beta=\beta_{1} \vee \cdots \vee \beta_{n}$, (ii) $\gamma=\gamma_{1} \vee \cdots \vee \gamma_{m}$, (iii) $\beta_{i}$ is not a join for any $i \in\{1, \ldots, n\}$, (iv) $\gamma_{j}$ is not a join for any $j \in\{1, \ldots, m\}$.

We proceed by induction on the pair $(n, m)$. If $n=1$ or $m=1$, the claim follows by Lemma 8. For inductive step, we have $\vdash \beta_{i} \Rightarrow \alpha, \gamma_{1}, \ldots, \gamma_{m}$ for all $i \in\{1, \ldots, n\}$, and $\vdash \gamma_{j} \Rightarrow \alpha, \beta_{1}, \ldots, \beta_{n}$ for all $j \in\{1, \ldots, m\}$. Applying Lemma 7, we get

$$
\begin{gathered}
\forall i \in\{1, \ldots, n\} \exists \tau \in\{\alpha\} \cup R^{b}(\gamma): \vdash \beta_{i} \Rightarrow \tau, \\
\forall j \in\{1, \ldots, m\} \exists \sigma \in\{\alpha\} \cup R^{b}(\beta): \vdash \gamma_{j} \Rightarrow \sigma .
\end{gathered}
$$

Thus, the joinands of $\beta$ can be partitioned into these that prove $\alpha$ and those that prove one of the joinands of $\gamma$. And similarly, the joinands of $\gamma$ can be partitioned into these that prove $\alpha$ and those that prove one of the joinands of $\beta$.

To make it precise, let us define $P \subseteq\{1, \ldots, n\}$, putting $i \in P$ if $\vdash \beta_{i} \Rightarrow \alpha$. Then $i \notin P$ if and only if $\vdash \beta_{i} \Rightarrow \gamma_{j}$ for some $j \in\{1, \ldots, m\}$. Put $\neg P=\{1, \ldots, n\} \backslash P$. Similarly, define $Q \subseteq\{1, \ldots, m\}$, by $j \in Q$ if $\vdash \gamma_{j} \Rightarrow \alpha$. Then $j \notin Q$ if and only if $\vdash \gamma_{j} \Rightarrow \beta_{i}$ for some $i \in\{1, \ldots, n\}$. Put $\neg Q=\{1, \ldots, m\} \backslash Q$.

Restating the above more formally, we have

$$
\begin{aligned}
& \forall i \in \neg P \exists j \in \neg Q: \vdash \beta_{i} \Rightarrow \gamma_{j}, \\
& \forall j \in \neg Q \exists i \in \neg P: \vdash \gamma_{j} \Rightarrow \beta_{i} .
\end{aligned}
$$

Now, assume $\vdash \beta_{i} \Rightarrow \gamma_{j}$ and $\vdash \gamma_{j} \Rightarrow \beta_{k}$ hold for some $i, j, k$ with $k \neq i$. Then, we get $\vdash \beta_{i} \Rightarrow \beta_{k}$ holds, and therefore $\vdash \beta \Leftrightarrow \bigvee R^{b}(\beta) \backslash\left\{\beta_{k}\right\}$. Let $\beta^{\prime}=\bigvee R^{b}(\beta) \backslash\left\{\beta_{k}\right\}$. Then, $\vdash \beta^{\prime} \Rightarrow \alpha, \gamma$ and $\vdash \gamma \Rightarrow \alpha, \beta^{\prime}$ hold, by Lemma 2 . Therefore, the inductive hypothesis applies to the pair $\left(\beta^{\prime}, \gamma\right)$ yielding $\vdash \beta^{\prime} \Rightarrow \alpha, \beta^{\prime} \wedge \gamma$, and then the required $\vdash \beta \Rightarrow \alpha, \beta \wedge \gamma$ follows by Lemma 2.

We can now assume that whenever $\vdash \beta_{i} \Rightarrow \gamma_{j}$ and $\vdash \gamma_{j} \Rightarrow \beta_{k}$ hold for some $i, j, k$, we have $k=i$. By symmetry, the same holds for any $i, j, k$ with $\vdash \gamma_{i} \Rightarrow \beta_{j}$ and $\vdash \beta_{j} \Rightarrow \gamma_{k}$. It follows that $|\neg P|=|\neg Q|$ via the bijection $f: P \rightarrow Q$ defined by $f(i)=j$ if $\vdash \beta_{i} \Leftrightarrow \gamma_{j}$. 
Next, we wish to split the join $\beta_{1} \vee \cdots \vee \beta_{n}$ into two parts: the part $\beta^{\prime}$ such that $\vdash \beta^{\prime} \Rightarrow \alpha$ holds, and $\beta^{\prime \prime}$ - the rest. We have to do it in a slightly roundabout fashion, since we do not have the easy option of defining $\bigvee \varnothing$ to be the bottom element. Therefore, for $X \in\{P, \neg P\}$ we put

$$
\beta_{X}= \begin{cases}\bigvee\left\{\beta_{i}: i \in X\right\} & \text { if } X \neq \varnothing \\ \text { undefined } & \text { if } X=\varnothing\end{cases}
$$

Similarly, for $Y \in\{Q, \neg Q\}$ we put

$$
\gamma_{Y}= \begin{cases}\bigvee\left\{\gamma_{j}: j \in Y\right\} & \text { if } Y \neq \varnothing \\ \text { undefined } & \text { if } Y=\varnothing\end{cases}
$$

Since $|\neg P|=|\neg Q|$, we have that $\beta_{\neg P}$ is defined if and only if $\gamma_{\neg Q}$ is. We will proceed assuming that both $\beta_{P}$ and $\beta_{\neg P}$ are defined, asking the reader to supply the (easy) details for the cases when one of them is not defined. Note that under our assumption $\vdash \beta_{P} \vee \beta_{\neg P} \Leftrightarrow \beta$ holds.

By definition of $\beta_{P}$, we have $\vdash \beta_{P} \Rightarrow \alpha$. Applying Lemma 2 several times to $\vdash \beta_{\neg P} \Rightarrow$ $\beta_{\neg P}$ we obtain $\vdash \beta_{\neg P} \Rightarrow \gamma_{\neg Q}$. Furthermore, from the definition of $\gamma_{\neg Q}$ it immediately follows that $\vdash \gamma_{\neg Q} \Rightarrow \gamma$ holds, and so we get $\vdash \beta_{\neg P} \Rightarrow \gamma$. Similarly, $\vdash \beta_{\neg P} \Rightarrow \beta$ follows from the definition of $\beta_{\neg P}$. Now we have the following valid proof:

$$
\frac{\frac{\beta_{P} \Rightarrow \alpha \quad}{\beta_{P} \Rightarrow \alpha, \beta \wedge \gamma} \quad \frac{\beta_{\neg P} \Rightarrow \beta \quad \beta_{\neg P} \Rightarrow \gamma}{\beta_{\neg P} \Rightarrow \beta \wedge \gamma}}{\beta_{\neg P} \Rightarrow \alpha, \beta \wedge \gamma}
$$

from which the required $\vdash \beta \Rightarrow \alpha, \beta \wedge \gamma$ follows by cut.

Lemma 10. Let $\alpha, \beta, \gamma$ be lattice terms. If $\alpha, \beta \Rightarrow \gamma$ and $\alpha, \gamma \Rightarrow \beta$ are provable, then so is $\alpha, \beta \vee \gamma \Rightarrow \beta$.

Proof. Follows from Lemma 9 by duality.

Theorem 4. Free lattices are semidistributive.

Proof. Assume $[\alpha] \vee[\beta]=[\alpha] \vee[\gamma]$ holds for some lattice terms $\alpha, \beta$ and $\gamma$. Then, we have $\vdash \alpha \vee \beta \Rightarrow \alpha \vee \gamma$ and thus, in particular, $\vdash \beta \Rightarrow \alpha, \gamma$ and $\vdash \gamma \Rightarrow \alpha, \beta$. By Lemma 9 we get $\vdash \beta \Rightarrow \alpha, \beta \wedge \gamma$. Therefore, $\vdash \alpha \vee \beta \Rightarrow \alpha \vee(\beta \wedge \gamma)$. Since $\vdash \alpha \vee(\beta \wedge \gamma) \Rightarrow \alpha \vee \beta$ holds as well, we obtain $[\alpha] \vee[\beta]=[\alpha] \vee([\beta] \wedge[\gamma])$, showing that $\left(\mathrm{SD}_{\vee}\right)$ holds. The proof of $\left(\mathrm{SD}_{\wedge}\right)$ uses Lemma 10 in an analogous way. 


\section{Two "logical" properties}

We finish by showing that the equational theory of lattices (equivalently, of free lattices) enjoys two properties that have probably never been considered in this context, because they are typically formulated with the help of an implication connective. The first of these is a form of interpolation, which we will dub order interpolation to distinguish it from many other versions of interpolation that have been investigated, especially in connection to amalgamation property in universal algebra (see [7] for one extensive study, or [13] for another, quite different in style). For a lattice term $\tau$ we let $V(\tau)$ stand for the set of variables occurring in $\tau$. We extend this notation to sets and multisets of terms in the obvious way. The property we will consider is the following:

- Let $\alpha$ and $\beta$ be lattice terms. If $\alpha \leqslant \beta$ holds in all lattices, then there exist a term $\tau$ such that $V(\tau) \subseteq V(\alpha) \cap V(\beta)$, and the inequalities $\alpha \leqslant \tau$ and $\tau \leqslant \beta$ hold in all lattices.

We will first employ a poor man's version of Maehara method (see, e.g., [16] for the fully fledged method, and [8] for an extended version) to prove the following lemma.

Lemma 11. Let $\Gamma$ and $\Delta$ be multisets of terms. If $\vdash \Gamma \Rightarrow \Delta$, then there exists a term $\tau$ such that $V(\tau) \subseteq V(\Gamma) \cap V(\Delta)$, and $\vdash \Gamma \Rightarrow \tau$ and $\vdash \tau \Rightarrow \Delta$ hold.

Proof. Consider a cut free proof $P$ of $\Gamma \Rightarrow \Delta$. Assume inductively that an interpolant exists for any proof shorter than $P$ (this trivially holds for proofs of length one). Now let $r$ be the last rule of $P$. We construct the interpolant $\tau$ case by case. For example, suppose $r$ is $\wedge$-introduction on the right, with $\Delta$ being the singleton $\{\alpha \wedge \beta\}$. By inductive assumption, we have terms $\varphi$ and $\psi$ such that $V(\varphi) \subseteq V(\Gamma) \cap V(\alpha), V(\psi) \subseteq V(\Delta) \cap V(\beta)$, and moreover we have $\vdash \Gamma \Rightarrow \varphi, \vdash \varphi \Rightarrow \alpha, \vdash \Gamma \Rightarrow \psi$, and $\vdash \psi \Rightarrow \beta$. Then, we immediately get $\vdash \Gamma \Rightarrow \varphi \wedge \psi$, and the following proof

$$
\frac{\frac{\varphi \Rightarrow \alpha}{\varphi \wedge \psi \Rightarrow \alpha} \frac{\psi \Rightarrow \beta}{\varphi \wedge \psi \Rightarrow \beta}}{\varphi \wedge \psi \Rightarrow \alpha \wedge \beta}
$$

showing that $\vdash \varphi \wedge \psi \Rightarrow \alpha \wedge \beta$ holds. In is easily checked that $V(\varphi \wedge \psi) \subseteq V(\Gamma) \cap V(\alpha, \beta)$, so $\tau=\varphi \wedge \psi$ is the required interpolant. All other cases are equally easy.

Putting $\Gamma=\{\alpha\}$ and $\Delta=\{\beta\}$ in Lemma 11, we obtain the next result as a corollary.

Theorem 5. The equational theory of lattices has the order interpolation property.

Since interpolation typically goes together with amalgamation, a word in this direction may be in order. It is well known that lattices have the amalgamation property. That is, for any V-formation $\left(L_{0}, L_{1}, L_{2}, i_{1}, i_{2}\right)$ where $L_{0}, L_{1}, L_{2}$ are lattices, and $i_{1}: L_{0} \rightarrow L_{1}, i_{1}: L_{0} \rightarrow L_{2}$ are embeddings, there exists an amalgam $\left(e_{1}, e_{2}, A\right)$, where $A$ is a lattice, and $e_{1}: L_{1} \rightarrow A$, $e_{2}: L_{2} \rightarrow A$ are embeddings such that the diagram below comutes. 


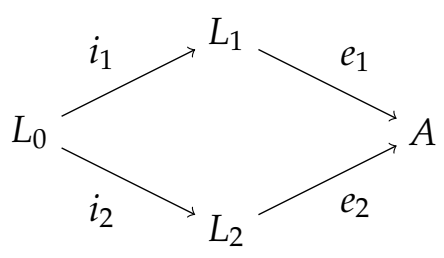

The amalgam is strong if $e_{1}\left(L_{1}\right) \cap e_{2}\left(L_{2}\right)=e_{1}\left(i_{1}\left(L_{0}\right)\right)=e_{2}\left(i_{2}\left(L_{2}\right)\right)$. Note that the second equality holds for any amalgam, it is the first one that makes it strong. For ordered structures, a further strengthening of amalgamation is useful, namely

- whenever $e_{1}(a) \leqslant e_{2}(b)$, there exists a $c \in L_{0}$ such that $a \leqslant i_{1}(c)$ and $i_{2}(c) \leqslant b$.

This property, known as superamalgamation, was first studied by Maximova in [12], and subsequently became one of the most investigated properties in algebraic logic (see e.g., [9, 10], also [7]). Superamalgamation is crucial for the existence of amalgamated free products of ordered structures (cf. [6] for lattices). It is easy to see that order interpolation is equivalent to superamalgamation of free lattices, that is, to the fact that the amalgamation diagram

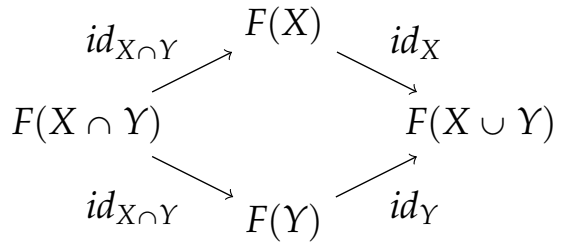

where $F(X \cap Y), F(X), F(Y)$ and $F(X \cap Y)$ are free lattices generated by sets $X$ and $Y$, such that $X \cap Y \neq \varnothing$, is a superamalgamation diagram. Indeed, it can be shown that this equivalence holds for any variety of ordered algebras, but we will not enter into details.

The last property we will consider is Maximova variable separation, first studied in [11]. In the present context it can be formulated as follows.

- Let $\alpha_{1}, \alpha_{2}, \beta_{1}, \beta_{2}$ be lattice terms such that $V\left(\alpha_{1}, \alpha_{2}\right) \cap V\left(\beta_{1}, \beta_{2}\right)=\varnothing$. If $\alpha_{1} \wedge \beta_{1} \leqslant \alpha_{2} \vee \beta_{2}$ holds in all lattices, then either $\alpha_{1} \leqslant \alpha_{2}$ holds in all lattices or $\beta_{1} \leqslant \beta_{2}$ holds in all lattices.

Theorem 6. Maximova variable separation holds for the equational theory of lattices.

Proof. The sequent translation of $\alpha_{1} \wedge \beta_{1} \leqslant \alpha_{2} \vee \beta_{2}$ is $\alpha_{1}, \beta_{1} \Rightarrow \alpha_{2}, \beta_{2}$, so assume we have $\vdash \alpha_{1}, \beta_{1} \Rightarrow \alpha_{2}, \beta_{2}$, with $V\left(\alpha_{1}, \alpha_{2}\right) \cap V\left(\beta_{1}, \beta_{2}\right)=\varnothing$. Applying Lemma 5 with $\Gamma=\left\{\alpha_{1}, \beta_{1}\right\}$ and $\Delta=\left\{\alpha_{2}, \beta_{2}\right\}$ we get that at least one of the following sequents

$$
\alpha_{1} \Rightarrow \alpha_{2} \quad \alpha_{1} \Rightarrow \beta_{2} \quad \beta_{1} \Rightarrow \alpha_{2} \quad \beta_{1} \Rightarrow \beta_{2}
$$

Australasian Journal of Logic (17:2) 2020, Article no. 2 
is provable. If either of the middle two is provable, then by Lemma 11 there is an interpolant $\tau$ such that $V(\tau) \subseteq V\left(\alpha_{i}\right) \cap V\left(\beta_{j}\right)$, where $\{i, j\}=\{1,2\}$. But, by assumption on the variables, we have $V\left(\alpha_{i}\right) \cap V\left(\beta_{j}\right)=\varnothing$. Since there are no constants in the signature, such an interpolant cannot exist, so neither of $\alpha_{1} \Rightarrow \beta_{2}, \beta_{1} \Rightarrow \alpha_{2}$ is provable. Thus, either $\alpha_{1} \Rightarrow \alpha_{2}$ or $\beta_{1} \Rightarrow \beta_{2}$ is provable, and thus $\alpha_{1} \leqslant \alpha_{2}$ holds or $\beta_{1} \leqslant \beta_{2}$ holds, as required.

Variable separation may play an auxiliary role in refining the naive decision algorithm. But this, as I already said, is another story.

\section{References}

[1] W. J. Blok and Don Pigozzi, Algebraizable logics, Mem. Amer. Math. Soc. 77 (1989), no. 396, vi+78.

[2] Josep Maria Font, Abstract algebraic logic, Studies in Logic (London), vol. 60, College Publications, London, 2016. An introductory textbook; Mathematical Logic and Foundations.

[3] Ralph Freese, Free lattice algorithms, Order 3 (1987), no. 4, 331-344.

[4] Ralph Freese, J. Ježek, and J. B. Nation, Term rewrite systems for lattice theory, J. Symbolic Comput. 16 (1993), no. 3, 279-288.

[5] George Grätzer, General lattice theory, 2nd ed., Birkhäuser Verlag, Basel, 1998. New appendices by the author with B.A. Davey, R. Freese, B. Ganter, M. Greferath, P. Jipsen, H.A. Priestley, H. Rose, E.T. Schmidt, S.E. Schmidt, F. Wehrung and R. Wille.

[6] G. Grätzer and A. P. Huhn, Amalgamated free product of lattices. I. The common refinement property, Acta Sci. Math. (Szeged) 44 (1982), no. 1-2, 53-66.

[7] Hitoshi Kihara and Hiroakira Ono, Interpolation properties, Beth definability properties and amalgamation properties for substructural logics, J. Logic Comput. 20 (2010), no. 4, 823-875.

[8] Tomasz Kowalski and Hiroakira Ono, Analytic cut and interpolation for bi-intuitionistic logic, Rev. Symb. Log. 10 (2017), no. 2, 259-283.

[9] Judit X. Madarász, Interpolation and amalgamation; pushing the limits. I, Studia Logica 61 (1998), no. 3, 311-345.

[10] _ Interpolation and amalgamation; pushing the limits. II, Studia Logica 62 (1999), no. 1, 1-19.

[11] L. L. Maksimova, The principle of separation of variables in propositional logics, Algebra i Logika 15 (1976), 168-184.

[12] _ Craig's theorem in superintuitionistic logics and amalgamable varieties of pseudo-Boolean algebras, Algebra i Logika 16 (1977), 643-681.

[13] George Metcalfe, Franco Montagna, and Constantine Tsinakis, Amalgamation and interpolation in ordered algebras, J. Algebra 402 (2014), 21-82.

[14] Hirokazu Nishimura, Proof theory for minimal quantum logic. II, Internat. J. Theoret. Phys. 33 (1994), no. 7, 1427-1443.

[15] _ Proof theory for minimal quantum logic. I, Proceedings of the International Quantum Structures Association, Part III (Castiglioncello, 1992), 1994, pp. 103-113.

[16] Hiroakira Ono, Proof theory and algebra in logic, Short Textbooks in Logic, Springer, Singapore, 2019.

[17] Don Pigozzi, Amalgamation, congruence-extension, and interpolation properties in algebras, Algebra Universalis 1 (1971/72), 269-349.

Australasian Journal of Logic (17:2) 2020, Article no. 2 